\title{
Environmental effects and risk control of antibiotic resistance genes in the organic solid waste aerobic composting system: A review
}

\author{
Caihong Huang ${ }^{1,2}$, Zhurui Tang ${ }^{1,2,3}$, Beidou Xi $(\bowtie)^{1,2,4}$, Wenbing Tan ${ }^{1,2}$, Wei Guo ${ }^{1,2}$, \\ Weixia $\mathrm{Wu}^{1,2,5}$, Caiyun $\mathrm{Ma}^{1,2,4}$
}

1 State Key Laboratory of Environmental Criteria and Risk Assessment, Chinese Research Academy of Environmental Sciences, Beijing 100012, China

2 Innovation Base of Ground Water \& Environmental System Engineering, Chinese Research Academy of Environmental Sciences, Beijing 100012, China

3 State Key Laboratory of Urban Water Resource and Environment, School of Environment, Harbin Institute of Technology, Harbin 150090, China 4 School of Environmental and Municipal Engineering, Lanzhou Jiaotong University, Lanzhou 730070, China

5 College of Environmental Science and Engineering, Guilin University of Technology, Guilin 541004, China

\section{H I G H L I G H T S}

- ARGs were detected in livestock manure, sludge, food waste and fermentation dregs.

- The succession of microbial community is an important factor affecting ARGs.

- Horizontal transfer mechanism of ARGs during composting should be further studied.

\section{A R T I C L E I N F O}

Article history:

Received 26 August 2020

Revised 18 February 2021

Accepted 19 February 2021

Available online 15 March 2021

\section{Keywords:}

Antibiotic resistance genes

Organic solid waste

Aerobic composting

Livestock manure

Sludge

\section{GR A P H I C A B T R A C T}

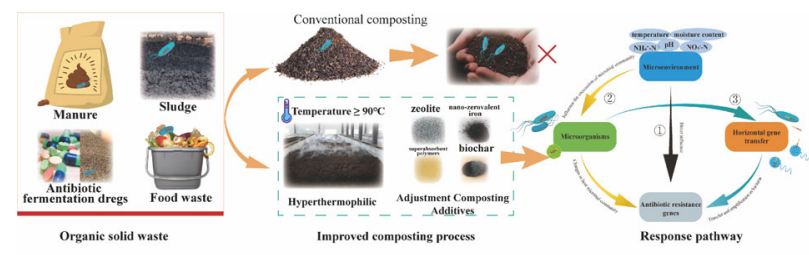

A B S T R A C T

Antibiotic resistance genes (ARGs) have been diffusely detected in several kinds of organic solid waste, such as livestock manure, sludge, antibiotic fermentation residues, and food waste, thus attracting great attention. Aerobic composting, which is an effective, harmless treatment method for organic solid waste to promote recycling, has been identified to also aid in ARG reduction. However, the effect of composting in removing ARGs from organic solid waste has recently become controversial. Thus, this article summarizes and reviews the research on ARGs in relation to composting in the past 5 years. ARGs in organic solid waste could spread in different environmental media, including soil and the atmosphere, which could widen environmental risks. However, the conventional composting technology had limited effect on ARGs removal from organic solid waste. Improved composting processes, such as hyperthermophilic temperature composting, could effectively remove ARGs, and the HGT of ARGs and the microbial communities are identified as vital influencing factors. Currently, during the composting process, ARGs were mainly affected by three response pathways, (I) "Microenvironment-ARGs"; (II) "Microenvironment-microorganisms-ARGs"; (III) "Microorganisms-horizontal gene transfer-ARGs", respectively. Response pathway II had been studied the most which was believed that microbial community was an important factor affecting ARGs. In response pathway III, mainly believed that MGEs played an important role and paid less attention to eARGs. Further research on the role and impact of eARGs in ARGs may be considered in the future. It aims to provide support for further research on environmental risk control of ARGs in organic solid waste.

(C) The Author(s) 2021. This article is published with open access at link.springer.com and journal.hep. com.cn 2021

\section{Introduction}

Antibiotic resistance genes (ARGs), as an emerging

$\triangle$ Corresponding author

E-mail: xibeidou@yeah.net contaminant, would enable microorganisms to acquire drug resistance, which had attracted widespread attention worldwide (Zhu et al., 2013; Manaia, 2017; Vikesland et al., 2017). ARGs have the characteristics of "easy to get, hard to lose" and tend to accumulate continuously in the environment (Mao et al., 2014; Dong et al., 2019). ARGs 
could transfer horizontally between microbial. If acquired by pathogenic bacteria, the production of "superbugs" would seriously threaten human health (Pruden et al., 2006; Martínez, 2008). China is the world's largest producer and user of antibiotics. In 2013, China used approximately 162000 tons of antibiotics, 52\% of which were veterinary antibiotics, and the surplus were medical antibiotics (Zhang et al., 2015). Veterinary antibiotics would eventually be residual in livestock feces, and municipal sludge often accumulates residual medical antibiotics (Karkman et al., 2018; Gurmessa et al., 2020). This phenomenon brings arduous challenges to the harmless treatment of organic solid waste.

Residual antibiotics, heavy metals, organic contaminants, and disinfectants in organic solid waste could also induce ARG production (Jia et al., 2015; Sun et al., 2015; Li et al., 2016; Yin et al., 2017). Although ARGs exist naturally in the environment, human activities, such as livestock and poultry breeding, pharmaceutical industry, and urban sewage treatment plants, have been determined to have greatly increased the spread of ARGs to the environment (Li et al., 2015; Karkman et al., 2018). ARGs in organic solid waste may especially diffuse into other environmental media through a series of ways, creating huge potential environmental health risks. Therefore, it is necessary to control ARGs in solid waste.

To reduce the spread of ARGs in organic solid waste, such as livestock manure and sludge, to the environment, the role of biological treatment technology in reducing ARGs has been emphasized. Aerobic composting is one of the main ways of harmless treatment and resource utilization of livestock manure and sludge (Chen et al., 2019b; Zhu et al., 2019a). Existing studies have proven that high-temperature aerobic composting can effectively remove various organic contaminants, such as antibiotics, and at the same time kill pathogenic bacteria (Zhang et al., 2019; Zhu et al., 2019a). In recent years, the use of composting technology to reduce ARGs in organic solid waste has become a research hotspot. Although most studies showed that composting can remove ARGs, some studies have demonstrated that composting can not effectively remove ARGs (Liao et al., 2018, 2019a; Tang et al., 2020). The removing effect of ARGs in compost still remains controversial. The changes in the abundance of ARGs during aerobic composting are often based on the combined results of multiple factors. The complicated microenvironmental changes of composting may be the important factors affecting ARGs. The changes in the compost microenvironment can often directly or indirectly affect ARGs as it also affects microorganisms. In addition, under the action of microorganisms, ARGs could increase abundance through horizontal gene transfer (HGT) or vertical gene transfer (VGT).

Although there had been numerous studies on ARGs in composting, due to different environmental conditions such as composting technology and materials, some opinions and conclusions were still relatively controversial, which hinders how to use composting technology to control the environmental risk of ARGs in organic solid waste. Based on this, this article summarizes the research on the removal of ARGs from organic solid waste composting in the past five years, trying to explain the potential environmental risks of ARGs in organic solid waste and their transmission routes across environmental media; discuss the three main response pathways of ARGs abundance changes during composting; summarize the improved composting process used to remove ARGs. Finally, existing research problems and future research are put forward as the focus. This article aims to provide a reference for the subsequent reduction and control of ARGs in organic solid waste.

\section{Environmental risks of ARGs in organic solid waste}

There was a global concern about the spread of ARGs, and their acquisition by clinically relevant microorganisms increases the hospitalization and mortality rate of patients, which poses a serious problem for the health and welfare of humans (Zhu et al., 2017). At least 700000 people die annually from antimicrobial-resistance infections (Li et al., 2018), and scientists predicted this number would mushrooming to 10 million by 2050 when antimicrobialresistance infections would become a more dangerous killer than cancer (O'Neill, 2014). Therefore, the ARGs and antimicrobial-resistance had been recognized by the world health organization as one of the biggest challenges of 21 st century.

The generation and increased diffusion of ARGs in the environment mainly give rise to three influencing mechanisms, including HGT, genetic mutation and recombination, and selective pressure of antimicrobial compounds or other contaminants, such as antibiotics, heavy metals, and subinhibitory concentrations of disinfectants (Berendonk et al., 2015). The dissemination of ARGs is mainly attributed to the ability of bacteria, including clinically relevant pathogens, to acquire genes through HGT. The three basic HGT mechanisms are transduction, conjugation, and transformation. Mobile genetic elements (MGEs), such as plasmids, integrons, or transposons, play a considerable role in HGT. ARGs can multiply with their hosts' amplification, and VGT can make ARGs persist in the environment.

ARGs are widely present in natural environments, including aerosols, soil, surface water, sewage, and oligotrophic Antarctic (Li et al., 2015; Zhang et al., 2015; Wang et al., 2016a; Li et al., 2020b; Zammit et al., 2020). However, the release of ARGs from anthropogenic sources has exacerbated ARG accumulation in the environment ( $\mathrm{Li}$ et al., 2015). Since the widespread use of antibiotics in 1940, ARGs have increased significantly 
(Charles et al., 2010). Antibiotics are divided into veterinary and medical antibiotics. Although the objects of use are different, they would eventually affect ARGs in organic solid waste. Parts of unused and expired medicines are usually discarded in municipal solid waste, and other human antibiotics eventually end up in the sewage (Karkman et al., 2018). In wastewater treatment plants, these residual antibiotics and subinhibitory concentrations of disinfectants would induce the generation of ARGs through selective pressure (Zhang et al., 2017b). Wastewater treatment plants have become an important reservoir of resistance genes (Karkman et al., 2018). In wastewater treatment plants, antibiotic resistance bacteria (ARB) and ARGs would enter the sludge as the treatment progresses. Veterinary antibiotics are commonly used in the livestock and poultry industry and animal husbandry. However, $50 \%-80 \%$ of antibiotics are excreted in the form of original or metabolites with feces and urine, resulting in large amounts of antibiotic residues in livestock and poultry feces (Jjemba, 2006). Residual antibiotics in animal manure would induce many ARGs (Zhang et al., 2017a). In addition, the residual antibiotic fermentation residues in the pharmaceutical industry also have also been identified to have a high abundance of ARGs (Liao et al., 2019b). These factors make organic solid waste a "hotbed" for pathogenic bacteria to obtain ARGs and provide a prerequisite for producing "superbugs". Therefore, ARGs in organic solid waste have potential environmental health risks.

ARGs in organic solid waste are transferred to other environmental media, such as aerosols, soil, and surface water, through a series of processes, causing the spread of
ARG environmental risks (Fig. 1). Antibiotic resistance bacteria (ARB) and ARGs are transferred with the disposal of organic solid waste, such as landfill, aerobic composting, anaerobic digestion, and incineration, consequently posing a threat to public health (Fang et al., 2015; Wu et al., 2017; Chen et al., 2019a; Li et al., 2020b). A typical transmission pathway of ARGs in organic solid waste is to enter the soil environment and then invade the food chain through soil organisms and crops, posing threats to human health. Organic solid waste is processed through aerobic composting to produce high-quality compost products. With the application of composting products, the nutrients, $\mathrm{ARB}$, and ARGs are carried into the soil environment (Fang et al., 2015). Notably, compared to the application of manure into the soil after composting, the direct application of manure without composting might increase the abundance of ARGs in the soil environment (Chen et al., 2019a). Biogas residues may return directly to the field or enter the soil environment after further treatment by aerobic composting and also carrying ARB and ARGs in the biogas residue. ARGs in the soil environment then enter the food chain through the predation behavior of soil organisms and absorption of plants (Zhu et al., 2018; Zhu et al., 2019b). In addition, landfill treatment of organic solid waste normally produces plenty of leachates. With years of increasing landfill, the ARG abundance in leachates increases, which can be attributed to the heavy metals remaining in old leachates (Wu et al., 2017). In some simple landfills, leachates could contaminate the groundwater and transfer the environmental risks of ARGs to the groundwater environment. In addition, livestock and poultry farming, one of the sources of organic solid waste,

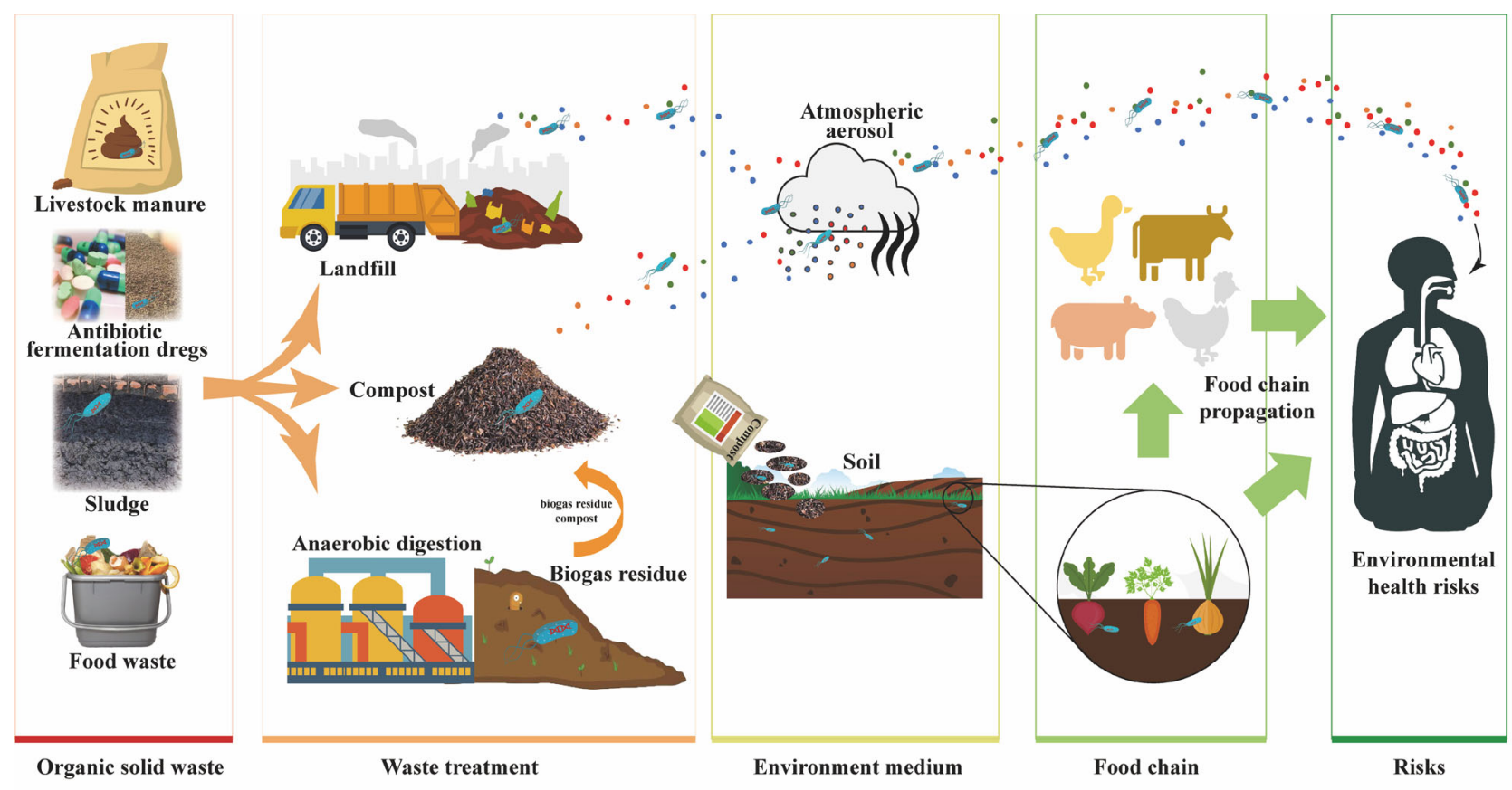

Fig. 1 Transmission of antibiotics resistant bacteria and antibiotics resistant genes in organic solid waste across environmental media. 
causes the input of ARGs to the soil and groundwater in livestock and poultry farms (Gao et al., 2020). Furthermore, landfill and incineration facilities promote ARGs in organic solid waste to diffuse into ambient air ( $\mathrm{Li}$ et al., 2020b). Recent studies have found that ARGs in compost also interact with ARGs in the surrounding atmosphere (Cui et al., 2020).

In organic solid waste, ARGs would be transferred out through a series of diffusion pathway in addition to their existing environmental risks, thus threatening the public health. Therefore, it is necessary to control ARGs in organic solid waste through an appropriate means.

\section{Removal mechanism and response path- way of ARGs in aerobic composting}

Aerobic composting, an important harmless treatment and resource utilization, promotes the conversion of organic matter into humic substances under the action of microorganisms and at the same time produces high-quality organic fertilizers (Xi et al., 2016; Yu et al., 2018). In composting, the main force of the microorganisms interacts with the compost microenvironment. Due to the high bacterial load in composting, residual organic contaminants, such as tylosin and other antibiotics, in organic solid waste are degraded and transformed through a series of biochemical reactions (Liao et al., 2019b). Furthermore, in aerobic conditions, the bacteria's enzyme system is thriving and active, which only accelerates contaminant degradation. Whether this harmless function could also be used to remove ARGs is yet unknown.
Recently, the removal of ARGs in organic solid waste through composting has become a research hotspot. This current research examining the effect of composting treatment on the removal of ARGs has both positive and opposing views, but the composting process certainly affects the ARG abundance changes (Zhang et al., 2017a; Liao et al., 2018; Chen et al., 2019a; Li et al., 2020a). In different composting studies, the abundance trend of ARGs is also different. Liao et al. (2019a) found that the absolute abundance of ARGs in composting increases with the composting process, and it cannot effectively reduce ARGs. However, Wang et al. (2016b) showed that the abundance of ARGs continues to decrease as composting progresses. This might be because ARGs are affected by many factors during the composting process. The composting system's environment in different studies has also been found to vary, presenting different trends on the abundance of ARGs during the composting process.

During the composting process, the generation and degradation of ARGs control the ARG abundance changes. The effects of these two behaviors are classified into three main factors: microenvironment, microorganisms, and HGT. These factors could affect ARGs directly through biochemical reactions or indirect response pathways. ARG abundance changes are the result of the combined effects of these factors. The three response pathways are divided into (I) "microenvironment-ARGs", (II) "microenvironment-microorganisms-ARGs", and (III) "microorganisms-HGT-ARGs" (Fig. 2). In pathway I, microenvironmental factors directly affect ARGs. Temperature is an important influencing factor in composting, and it can significantly vary during the composting

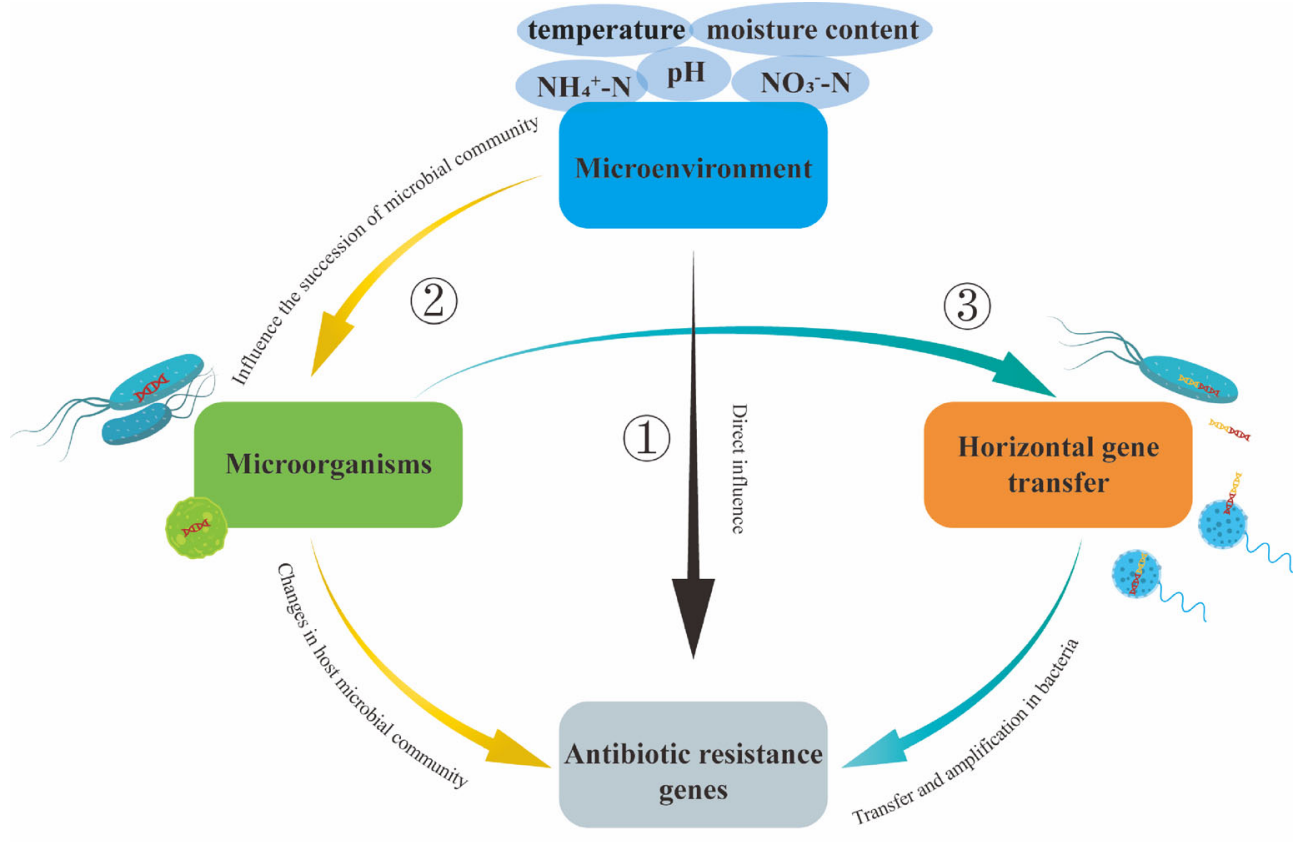

Fig. 2 Three main ARGs response pathways in compost. (1) "Microenvironment-ARGs"; (2) "Microenvironment-microorganismsARGs"; (3) "Microorganisms-HGT-ARGs". 
process. The temperature range of the traditional composting process in the thermophilic phase is from $50^{\circ} \mathrm{C}$ to $75^{\circ} \mathrm{C}$, but the compost pile temperature increases to above $90^{\circ} \mathrm{C}$ in hyperthermophilic composting processes (Liao et al., 2018). Extracellular DNA or plasmids derived from microbial cell lysis at high temperatures also contain ARGs and/or MGEs (Nielsen et al., 2007a; Liao et al., 2019a), and these extracellular DNAs can survive even at $70^{\circ} \mathrm{C}$ (Zhang and $\mathrm{Wu}, 2005$ ). However, at higher temperatures, such as above $85^{\circ} \mathrm{C}$, DNA is degraded into much smaller pieces (Zhang and $\mathrm{Wu}, 2005)$. In addition, other microenvironments, such as material particle size and dissolved organic matter (DOM), directly affect ARGs. In compost, there are few studies on the material particle size and DOM directly affecting ARGs. However, in other environmental media, related studies have been reported (Zhang et al., 2019b; Lian et al., 2020). For example, Lian et al. found that both bulk-biochar and nano-biochar can adsorb ARGs, whereas nano-biochar can degrade ARGs by generating persistent free radicals (Lian et al., 2020). DOM produces hydroxyl radicals through photosensitization, which could degrade extracellular ARGs (eARGs; Zhang et al., 2019b). These studies in different environmental media might provide a reference for further research on the impact of the compost microenvironment on ARGs.

The interaction between the microenvironment and microorganisms is an important driving force for compost maturation, and this process affects the ARG abundance changes. The "microenvironment-microorganisms-ARGs" pathway is currently gaining huge attention in the composting process. In this response pathway, the microenvironment affects the microbial properties and community structure (Wang et al., 2016b; Guo et al., 2019b; Liao et al., 2019b). The microenvironment directly affects the microbial properties, such as microbial activity, cellular SOS response, and microbial community change, which then affects ARGs. Moisture content is one of the important environmental parameters of composting, as microbial activity is closely related to it. Therefore, changes in moisture content affect the abundance of ARGs by altering the activity of host microorganisms (Guo et al., 2017; Cheng et al., 2019; Liao et al., 2019a). Higher $\mathrm{pH}$ also causes SOS response, promotes ARG transfer, and increases ARG abundance (Ren et al., 2019). The result that the microenvironment affects the microbial properties and changes ARG abundance is more of a speculation. High-throughput sequencing and statistical analysis revealed that, with a change of the microenvironment, the microbial community structure is also different, as the abundance of ARGs shows varying trends in different stages of composting. Composting is a dynamic process dependent on the combined activities of a wide variety of microbial populations and linked to the microenvironment. According to previous studies, these microenvironmental parameters include moisture content, temperature, $\mathrm{pH}$, total nitrogen, organic matter, $\mathrm{NH}_{4}{ }^{+}-\mathrm{N}$, and $\mathrm{NO}_{3}{ }^{-} \mathrm{N}(\mathrm{Su}$ et al., 2015; Yin et al., 2017; Qian et al., 2018; Cao et al., 2020; Chang et al., 2020; Fan et al., 2020; Li et al., 2020a). For example, in co-composting of swine manure and spent mushroom substrate, environmental parameters explained $37.66 \%$ of ARG profiles, and the water-soluble carbon, $\mathrm{NH}_{4}{ }^{+}-\mathrm{N}$, and $\mathrm{NO}_{3}{ }^{-}-\mathrm{N}$ had significant positive correlations in the top 35 genera (Hu et al., 2019). Hu et al. suggested that the contributions of environmental parameters to ARG profiles are mainly due to their effects on potential hosts. Similarly, during co-composting of swine manure and cotton stalks, environmental parameters, such as moisture content, temperature, $\mathrm{pH}, \mathrm{NH}_{4}{ }^{+}-\mathrm{N}$, and $\mathrm{NO}_{3}{ }^{-}-\mathrm{N}$, explained $42.6 \%$ and $78.4 \%$ variation in ARG profiles and changes in the potential host bacteria, respectively (Zhang et al., 2018b). These results showed that the variations in ARGs were determined more by the dynamics of the potential host bacteria during composting (Zhang et al., 2016b, 2017a, 2018a). However, in some other studies, different set of physicochemical parameters that mainly contribute to the change of ARGs were identified (Guo et al., 2019a; Peng et al., 2020). This might be due to the growth and incubation of potential host microorganisms carrying ARGs affected by multiple physicochemical parameters (Wang et al., 2020b). With the changes in the composting system and original materials, the environmental factors that might have strongly influenced ARGs in compost were different. Subsequent research might need to find out the strong influencing factors for a certain type of composting original materials or system to realize the regulation mechanism of "microenvironment-microorganisms-ARGs".

In response pathway III, ARGs carried by microorganisms diffuse through HGT, affecting the abundance of ARGs. MGEs, such as integrons, plasmids, and transposons, play critical roles in transferring ARGs among bacteria. At present, MGEs associated with ARGs in compost are more concerned about integrons (intll, intl2, and ISCR1), transposons (Tn916/1545, Tn914/1545, IS613, and tnpA), and plasmids (IncQ and oriV; Zhang et al., 2016a; Liu et al., 2018b; Qian et al., 2018; Cao et al., 2020). The relationship among ARGs, MGEs, and microorganisms in compost is generally based on the following assumptions: ARGs might have been integrated into integrons; afterward, integrons with ARGs are further configured into transposons or plasmids, and then, the microorganisms accept ARGs by conjugation and transformation with transposons or plasmids. In previous studies on MGEs and ARGs in compost, an MGE might affect the abundance changes of multiple ARGs. Wang et al. (2019) found that the abundance of intl1 is positively correlated with $t e t W$, tet $O$, sull, and sul2, which may be due to the integration of these ARGs into Intl1 and HGT among different microorganisms during composting. Similarly, Liu et al. (2018b) showed that the five resistance genes of aminoglycosides are significantly related to MGEs, and they believed that some aminoglycosides 
might have undergone HGT through the action of MGEs. The HGT of ARGs with MGEs might also enable some pathogenic bacteria to obtain ARGs, increasing environmental risks. Studies have shown that class 1 integrons (Intl1) in MGEs could carry a gene cassette, including multiple ARGs for HGT (Ma et al., 2017). In addition, the contribution of eARGs in the HGT process should also be considered. At present, research on eARGs mainly focuses on livestock and poultry breeding wastewater treatment (Zhang et al., 2013), urban lake sediments (Dong et al., 2019), and urban domestic sewage treatment, among other aspects (Liu et al., 2018a; Zhang et al., 2018c; Che et al., 2019; Zhou et al., 2019). Recently, studies on eARGs in anaerobic fermentation have also been reported, and eARGs accounted for $1.5 \%-86.5 \%$ of the total ARGs (Zou et al., 2020). The sources of eARGs include the lysis of dead cells and the secretion of live cells. Studies have shown that a high microbial abundance environment is like a dynamic gene pool of eARGs, and bacteria could naturally transform from this gene pool (Cai et al., 2006). This implies that eARGs with a stronger HGT capacity have greater potential environmental risks because pathogenic bacteria may more likely come into contact with eARGs. In composting, due to the fierce succession of the microbial community, the rapid death of bacteria may accelerate the accumulation of eARGs, which is an important factor in the composting process. Therefore, whether the release of eARGs would be accelerated in the biotransformation process of organic solid waste with more frequent and complex microbial community succession remains to be unknown, and how to promote the HGT process of ARGs is yet to be determined. Further research on these issues would help in understanding the removal of ARGs in compost.

\section{Improving conventional composting enhances degradation efficiency of ARGs}

The traditional organic solid waste treatment process seems to be difficult in terms of coping with the environmental risks of ARGs (Su et al., 2015; Youngquist et al., 2016). More and more studies have tried to enhance the removal efficiency of ARGs by improving the traditional organic solid waste treatment process. In terms of composting technology, some studies have promoted the reduction of ARGs by regulating the composting temperature, adjusting composting additives, and using semi-permeable membrane-covered thermophilic aerobic composting system.

\subsection{Hyperthermophilic composting}

Temperature is considered as one of the most vital environmental parameters in composting. In the thermo- philic phase of composting, the temperature range is usually from $50^{\circ} \mathrm{C}$ to $70^{\circ} \mathrm{C}$. In this composting phase, ARGs generally also have higher removal efficiency (Wang et al., 2015). Based on this feature, Liao et al. used hyperthermophilic composting technology to improve the ARG removal effect in compost (Liao et al., 2018). The hyperthermophilic composting technology was first developed by Oshima (Oshima and Moriya, 2008). This composting technology can raise the maximum temperature of composting to about $90^{\circ} \mathrm{C}$ without external heating, which is $20^{\circ} \mathrm{C}-30^{\circ} \mathrm{C}$ higher than traditional composting. Liao et al. selected extreme thermophilic bacteria from environmental samples, such as the mountain pass (Tengchong crater in Yunnan, China) and hot springs (hot springs in Zengcheng, Guangdong, China), and used them in the composting process (Moriya et al., 2011; Yu et al., 2015).

Compared to traditional thermophilic aerobic composting (the control group), hyperthermophilic temperature composting had a higher ARG removal rate (89\%). Especially in the hyperthermophilic temperature phase of composting, the removal rate was even as high as $91 \%$. Although the abundance of ARGs increased slightly during the maturity stage of composting, it was still lower than the abundance of ARGs in traditional composting. It is worth noting that the removal rate of MGEs by hyperthermophilic temperature composting was $49 \%$, which is considered lower than the removal rate of ARGs but higher than traditional composting. Furthermore, the half-life of ARGs and MGEs in hyperthermophilic temperature composting was 1.3 days, much lower than the 4.0 days of traditional composting. Based on the partial least-squares path model analysis results, the reduction of ARGs had different response mechanisms in hyperthermophilic temperature composting and traditional composting. In hyperthermophilic temperature composting, the change in the abundance of MGEs is a strong influencing factor of ARGs. The reduction of ARG abundance may be due to the reduction of MGEs, which inhibits the HGT process of ARGs, (response pathway III: "microorganisms-HGT-ARGs"). In traditional composting, the changes in the main microbial communities can affect the abundance of ARGs (response pathway II). In general, hyperthermophilic temperature composting has proven to be an effective technique in reducing ARGs.

\subsection{Adjustment composting additives}

Composting materials include organic solid waste, such as livestock manure, sludge, and municipal solid waste, and composting additives, such as straw and wood chips. Composting additives often adjust the moisture content and carbon/nitrogen ratio and improve the aeration conditions and other effects, thereby promoting compost maturity. Therefore, some studies have tried to promote the reduction of ARGs by regulating composting additives. As 


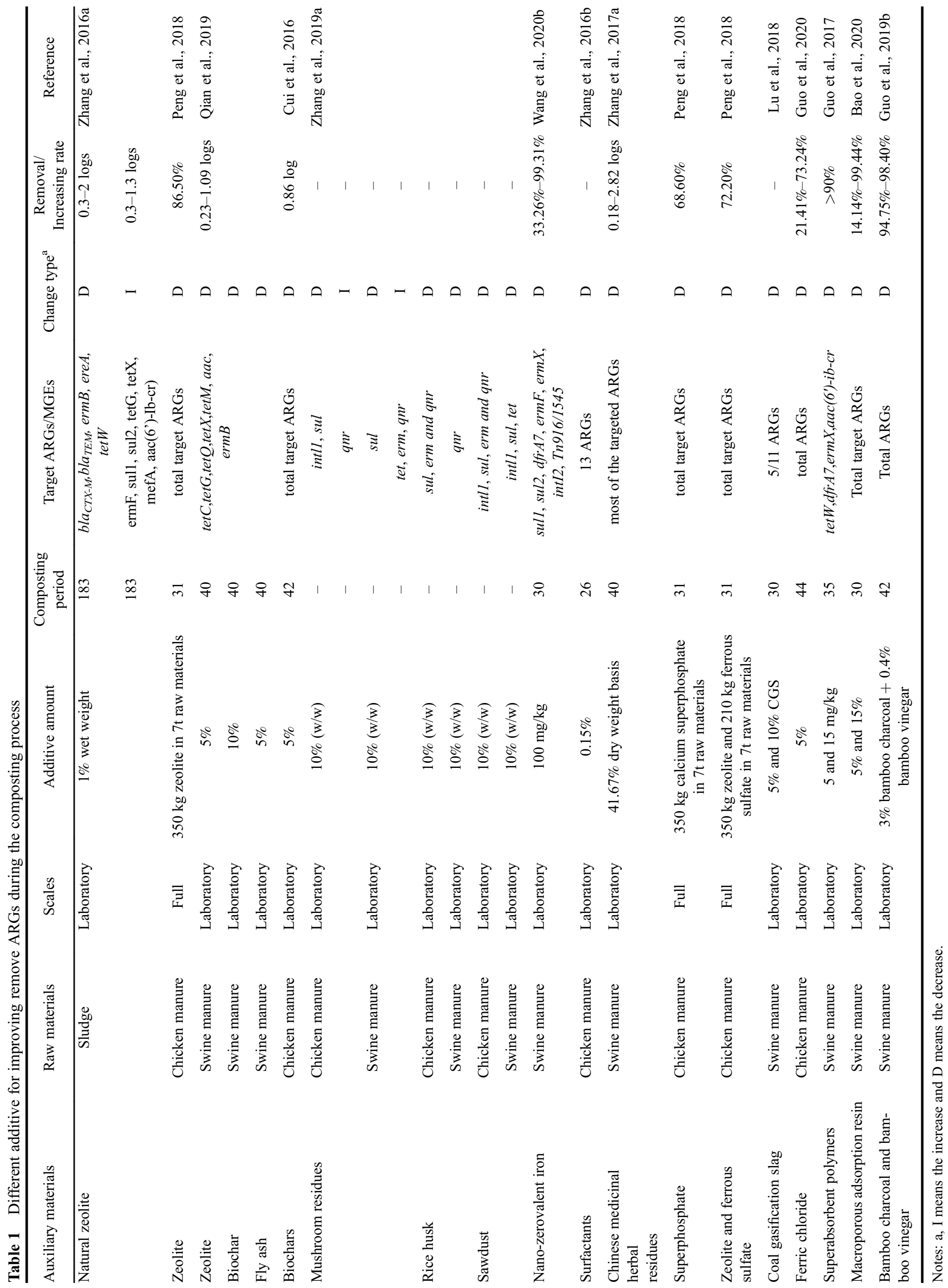


shown in Table 1, the additives include zeolite, biochar, and nano-zerovalent iron, and composting materials mainly include sludge and livestock manure. The addition of auxiliary materials has achieved effective results in the removal of ARGs. Most of the research on the addition and removal of ARGs in composting additives concentrated on the laboratory scale. Experiments on the industrial scale include the addition of zeolite, ferrous sulfate, and superphosphate, and the removal efficiency of ARGs reached $68.6 \%-86.5 \%$. This shows that adding auxiliary materials to promote the removal of ARGs has certain industrial feasibility. However, it is worth noting that the addition of some auxiliary composting materials can bring in varying effects, depending on the type of ARGs. In the sludge compost, with the addition of $1 \%$ natural zeolite, the abundance of total ARGs decreased by $1.5 \%$; a further classification into ARG types found that the abundance of seven ARGs increased, and only five ARGs decreased (Zhang et al., 2016a). Similarly, Lu et al. (2018) found that in pig manure compost with fly ash, only 5 of the 11 target ARGs were reduced in terms of abundance. This means that the removal efficiency of different ARG types varies depending on the auxiliary materials added. This might be due to the different response mechanisms of different additives to ARGs in composting. For example, Wang et al. (2020a) found that the tetracycline adsorbed by biochar through the $\mathrm{H}$-bonding of oxygen-containing functional groups on the surface still had high bioavailability, which caused selective pressure on Escherichia coli and led tetM to be expressed in large quantities.

The addition of auxiliary materials in the compost would change the microenvironmental parameters, such as porosity, moisture content, nutrient level, and $\mathrm{pH}$ value. These changes in the microenvironment could indirectly affect the changes in ARGs as it affects microorganisms and MGEs. Qian et al. (2019) added biochar, fly ash, and zeolite to promote ARG removal in pig manure compost. In this study, changes in the bacterial community determined the changes in the abundances of ARGs as demonstrated by redundancy analysis. Moreover, the inconsistent effects of the additives, such as biochar, fly ash, and zeolite on ARGs, can be attributed to their varying influence on bacterial composition. The addition of biochar significantly decreased the hosts of sul2; thus, biochar was more effective in reducing the abundance of sul2 than zeolite and fly ash. Biochar may be beneficial to the colonization of microorganisms because its particle size scale is suitable for spores, bacteria, and other microbial communities (Ngo et al., 2013). However, some studies believed that zeolite's porous structure could reduce the contact rate between microorganisms; thus, HGT through conjugation was reduced (Zhang et al., 2016a). Some studies also reported that the addition of auxiliary materials, such as bamboo charcoal and bamboo vinegar, reduces MGEs, thereby controlling ARG abundance (Guo et al., 2019b). In summary, although adding different auxiliary materials could effectively reduce the abundance of ARGs, the response mechanisms of additives to ARGs are still mainly response pathways II and III.

\subsection{Other improved composting processes and ARGs control prospects}

Traditional thermophilic aerobic composting is usually run in an open system, with a huge impact on the surrounding environment via the production of particulate matter and odor emissions (Gaviria-Figueroa et al., 2019). ARGs could exist on aerosol particles and spread in the air. Studies have shown that the composting plant interacts with ARGs in atmospheric particles around the plant (Gao et al., 2017). Based on this, Cui et al. (2020) adopted the new composting system, the semi-permeable membranecovered thermophilic aerobic composting system, which uses a semi-permeable membrane to cover and seal the compost material. The system would reduce the impact on the surrounding atmosphere and, at the same time, prevent particulate matter, such as bioaerosols, in the atmosphere from being transferred to the compost product. Through this semi-permeable membrane-covered composting system, ARGs and MGEs were reduced by $42.1 \%$ and $38.1 \%$. The abundance and diversity of ARB in the samples at the maturity stage of the composting system were also lower than that of the traditional thermophilic aerobic composting. This new type of composting process prevents the transfer of ARGs between aerosols and organic solid waste, thereby controlling the risk of ARGs in compost products.

The current research on the reduction mechanism of ARGs in compost was mainly based on the change of the microbial community and the removal of MGEs, which then affects the abundance of ARGs. In the process of microbial community changes, the change of host microorganisms is the key to removing ARGs. After the host microorganisms die, the cells are lysed, and the carried ARGs would flow out of the cell. Some of these eARGs would be degraded, whereas other ARGs can still exist in the environment for some time (Nielsen et al., 2007b). As for the response to the removal of ARGs by controlling the HGT, the main concern is the impact of changes in MGEs on ARGs. eARGs also play an important role in the HGT process, but the current research on removing ARGs from organic solid waste through composting has rarely discussed the role of eARGs. There are related studies on eARGs in other environmental media, such as livestock and poultry farm wastewater, domestic wastewater, and pharmaceutical factory wastewater (Dong et al., 2019; Sui et al., 2019; Yuan et al., 2019). The study of eARGs in compost can refer to related studies in other environmental media, providing new ideas for controlling ARGs by inhibiting the HGT pathway. 


\section{Conclusions and areas of focus for further research}

Compared to the traditional composting process, the improved composting process has been determined to effectively remove ARGs. The ARG removal mechanism in the composting process was mainly through three different response pathways, among which the HGT of microbial communities and ARGs were the main factors that directly affected ARGs. To further control the environmental risks of ARGs in organic solid waste, the following research can be considered: 1) source controlreduce the generation and accumulation of ARGs from the source of organic solid waste, such as strengthening the control of the use of antibiotics in livestock and poultry breeding and controlling the risk at the front end; 2) controlling the transmission route - expand the spread of ARGs from organic solid waste to other environmental media, such as water, soil, air, and other environmental media, and control technology research and control the risk of ARGs in organic solid waste from spreading through other channels; 3) control mechanism in composting carry out research on the ARG concentration level and the relationship between antibiotics-ARGs dose and effect and further elaborate the abundance change mechanism of ARGs during composting; 4) transfer mechanism of ARGs - the current research on resistance genes found in compost mainly focuses on the analysis of influencing factors, whereas the mechanism of ARG transfer among different microorganisms has been given less attention; and 5) ARG environmental risk assessment-with the progress of garbage classification and the construction of "zero-waste cities", the risk assessment index system of ARGs in organic solid waste needs to be improved.

Acknowledgements This study was supported by the National Key Research and Development Program of China (Grant No. 2019YFC1906401) and the Open Project of State Key Laboratory of Urban Water Resources and Environment, Harbin Institute of Technology, China (No. QAK201904).

Open Access This article is licensed under a Creative Commons Attribution 4.0 International License, which permits use, sharing, adaptation, distribution and reproduction in any medium or format, as long as you give appropriate credit to the original author(s) and the source, provide a link to the Creative Commons licence, and indicate if changes were made. The images or other third party material in this article are included in the article's Creative Commons licence, unless indicated otherwise in a credit line to the material. If material is not included in the article's Creative Commons licence and your intended use is not permitted by statutory regulation or exceeds the permitted use, you will need to obtain permission directly from the copyright holder. To view a copy of this licence, visit http://creativecommons.org/licenses/by/4.0/.

\section{References}

Bao J, Wang X, Gu J, Dai X, Zhang K, Wang Q, Ma J, Peng H (2020). Effects of macroporous adsorption resin on antibiotic resistance genes and the bacterial community during composting. Bioresource Technology, 295: 121997

Berendonk T U, Manaia C M, Merlin C, Fatta-Kassinos D, Cytryn E, Walsh F, Bürgmann H, Sørum H, Norström M, Pons M N, Kreuzinger N, Huovinen P, Stefani S, Schwartz T, Kisand V, Baquero F, Martinez J L (2015). Tackling antibiotic resistance: The environmental framework. Nature Reviews. Microbiology, 13(5): 310-317

Cai P, Huang Q Y, Zhang X W (2006). Interactions of DNA with clay minerals and soil colloidal particles and protection against degradation by DNase. Environmental Science \& Technology, 40(9): 29712976

Cao R, Wang J, Ben W, Qiang Z (2020). The profile of antibiotic resistance genes in pig manure composting shaped by composting stage: Mesophilic-thermophilic and cooling-maturation stages. Chemosphere, 250: 126181

Chang J, Jiang T, Zhao M, Yang J, Wen Z, Yang F, Ma X, Li G (2020). Variation pattern of antibiotic resistance genes and microbial community succession during swine manure composting under different aeration strategies. Journal of Chemical Technology and Biotechnology (Oxford, Oxfordshire), 95(2): 466-473

Charles W K, Jan D, Phillip A I E, Davidw G (2010). Evidence of increasing antibiotic resistance gene abundances in archived soils since 1940. Environmental Science \& Technology, 44(2): 580-587

Che Y, Xia Y, Liu L, Li A D, Yang Y, Zhang T (2019). Mobile antibiotic resistome in wastewater treatment plants revealed by Nanopore metagenomic sequencing. Microbiome, 7(1): 44

Chen C, Pankow C A, Oh M, Heath L S, Zhang L, Du P, Xia K, Pruden A (2019a). Effect of antibiotic use and composting on antibiotic resistance gene abundance and resistome risks of soils receiving manure-derived amendments. Environment International, 128: 233243

Chen X, Zhao Y, Zeng C, Li Y, Zhu L, Wu J, Chen J, Wei Z (2019b). Assessment contributions of physicochemical properties and bacterial community to mitigate the bioavailability of heavy metals during composting based on structural equation models. Bioresource Technology, 289: 121657

Cheng D, Feng Y, Liu Y, Xue J, Li Z (2019). Dynamics of oxytetracycline, sulfamerazine, and ciprofloxacin and related antibiotic resistance genes during swine manure composting. Journal of Environmental Management, 230: 102-109

Cui E, Wu Y, Zuo Y, Chen H (2016). Effect of different biochars on antibiotic resistance genes and bacterial community during chicken manure composting. Bioresource Technology, 203: 11-17

Cui P, Bai Y, Li X, Peng Z, Chen D, Wu Z, Zhang P, Tan Z, Huang K, Chen Z, Liao H, Zhou S (2020). Enhanced removal of antibiotic resistance genes and mobile genetic elements during sewage sludge composting covered with a semi-permeable membrane. Journal of Hazardous Materials, 396: 122738

Dong P, Wang H, Fang T, Wang Y, Ye Q (2019). Assessment of extracellular antibiotic resistance genes (eARGs) in typical environmental samples and the transforming ability of eARG. Environment International, 125: 90-96

Fan H, Wu S, Woodley J, Zhuang G, Bai Z, Xu S, Wang X, Zhuang X (2020). Effective removal of antibiotic resistance genes and potential links with archaeal communities during vacuum-type composting 
and positive-pressure composting. Journal of Environmental Sciences-China, 89: 277-286

Fang H, Wang H, Cai L, Yu Y (2015). Prevalence of antibiotic resistance genes and bacterial pathogens in long-term manured greenhouse soils as revealed by metagenomic survey. Environmental Science \& Technology, 49(2): 1095-1104

Gao F Z, Zou H Y, Wu D L, Chen S, He L Y, Zhang M, Bai H, Ying G G (2020). Swine farming elevated the proliferation of Acinetobacter with the prevalence of antibiotic resistance genes in the groundwater. Environment International, 136: 105484

Gao M, Jia R, Qiu T, Han M, Wang X (2017). Size-related bacterial diversity and tetracycline resistance gene abundance in the air of concentrated poultry feeding operations. Environmental Pollution, 220: $1342-1348$

Gaviria-Figueroa A, Preisner E C, Hoque S, Feigley C E, Norman R S (2019). Emission and dispersal of antibiotic resistance genes through bioaerosols generated during the treatment of municipal sewage. Science of the Total Environment, 686: 402-412

Guo A, Gu J, Wang X, Zhang R, Yin Y, Sun W, Tuo X, Zhang L (2017). Effects of superabsorbent polymers on the abundances of antibiotic resistance genes, mobile genetic elements, and the bacterial community during swine manure composting. Bioresource Technology, 244(Pt 1): 658-663

Guo H, Gu J, Wang X, Nasir M, Yu J, Lei L, Wang Q (2020). Elucidating the effect of microbial inoculum and ferric chloride as additives on the removal of antibiotic resistance genes from chicken manure during aerobic composting. Bioresource Technology, 309: 122802

Guo H, Gu J, Wang X, Tuo X, Yu J, Zhang R (2019a). Key role of cyromazine in the distribution of antibiotic resistance genes and bacterial community variation in aerobic composting. Bioresource Technology, 274: 418-424

Guo H, Gu J, Wang X, Yu J, Nasir M, Peng H, Zhang R, Hu T, Wang Q, Ma J (2019b). Responses of antibiotic and heavy metal resistance genes to bamboo charcoal and bamboo vinegar during aerobic composting. Environmental Pollution, 252: 1097-1105

Gurmessa B, Pedretti E F, Cocco S, Cardelli V, Corti G (2020). Manure anaerobic digestion effects and the role of pre- and post-treatments on veterinary antibiotics and antibiotic resistance genes removal efficiency. Science of the Total Environment, 721: 137532

Hu T, Wang X, Zhen L, Gu J, Zhang K, Wang Q, Ma J, Peng H (2019). Effects of inoculation with lignocellulose-degrading microorganisms on antibiotic resistance genes and the bacterial community during cocomposting of swine manure with spent mushroom substrate. Environmental Pollution, 252: 110-118

Jia S, Shi P, Hu Q, Li B, Zhang T, Zhang X X (2015). Bacterial community shift drives antibiotic resistance promotion during drinking water chlorination. Environmental Science \& Technology, 49(20): 12271-12279

Jjemba P K (2006). Excretion and ecotoxicity of pharmaceutical and personal care products in the environment. Ecotoxicology and Environmental Safety, 63(1): 113-130

Karkman A, Do T T, Walsh F, Virta M P J (2018). Antibiotic-resistance genes in waste water. Trends in Microbiology, 26(3): 220-228

Li B, Yang Y, Ma L, Ju F, Guo F, Tiedje J M, Zhang T (2015). Metagenomic and network analysis reveal wide distribution and cooccurrence of environmental antibiotic resistance genes. ISME
Journal, 9(11): 2490-2502

Li D, Zeng S, He M, Gu A Z (2016). Water disinfection byproducts induce antibiotic resistance-role of environmental pollutants in resistance phenomena. Environmental Science \& Technology, 50 (6): 3193-3201

Li H, Cheng W, Li B, Xu Y, Zheng X (2020a). The fate of antibiotic resistance genes during co-composting of swine manure with cauliflower and corn straw. Bioresource Technology, 300: 122669

Li L, Wang Q, Bi W, Hou J, Xue Y, Mao D, Das R, Luo Y, Li X (2020b). Municipal solid waste treatment system increases ambient airborne bacteria and antibiotic resistance genes. Environmental Science \& Technology, 54(7): 3900-3908

Li L G, Yin X, Zhang T (2018). Tracking antibiotic resistance gene pollution from different sources using machine-learning classification. Microbiome, 6(1): 93

Lian F, Yu W, Zhou Q, Gu S, Wang Z, Xing B (2020). Size matters: nano-biochar triggers decomposition and transformation inhibition of antibiotic resistance genes in aqueous environments. Environmental Science \& Technology, 54(14): 8821-8829

Liao H, Friman V P, Geisen S, Zhao Q, Cui P, Lu X, Chen Z, Yu Z, Zhou $S$ (2019a). Horizontal gene transfer and shifts in linked bacterial community composition are associated with maintenance of antibiotic resistance genes during food waste composting. Science of the Total Environment, 660: 841-850

Liao H, Lu X, Rensing C, Friman V P, Geisen S, Chen Z, Yu Z, Wei Z, Zhou S, Zhu Y (2018). Hyperthermophilic composting accelerates the removal of antibiotic resistance genes and mobile genetic elements in sewage sludge. Environmental Science \& Technology, 52(1): 266-276

Liao H, Zhao Q, Cui P, Chen Z, Yu Z, Geisen S, Friman V P, Zhou S (2019b). Efficient reduction of antibiotic residues and associated resistance genes in tylosin antibiotic fermentation waste using hyperthermophilic composting. Environment International, 133: 105203

Liu S S, Qu H M, Yang D, Hu H, Liu W L, Qiu Z G, Hou A M, Guo J, Li J W, Shen Z Q, Jin M (2018a). Chlorine disinfection increases both intracellular and extracellular antibiotic resistance genes in a fullscale wastewater treatment plant. Water Research, 136: 131-136

Liu Y, Feng Y, Cheng D, Xue J, Wakelin S, Li Z (2018b). Dynamics of bacterial composition and the fate of antibiotic resistance genes and mobile genetic elements during the co-composting with gentamicin fermentation residue and lovastatin fermentation residue. Bioresource Technology, 261: 249-256

Lu C, Gu J, Wang X, Liu J, Zhang K, Zhang X, Zhang R (2018). Effects of coal gasification slag on antibiotic resistance genes and the bacterial community during swine manure composting. Bioresource Technology, 268: 20-27

Ma L, Li A D, Yin X L, Zhang T (2017). The prevalence of integrons as the carrier of antibiotic resistance genes in natural and man-made environments. Environmental Science \& Technology, 51(10): 57215728

Manaia C M (2017). Assessing the risk of antibiotic resistance transmission from the environment to humans: non-direct proportionality between abundance and risk. Trends in Microbiology, 25(3): 173-181

Mao D, Luo Y, Mathieu J, Wang Q, Feng L, Mu Q, Feng C, Alvarez P J J 
(2014). Persistence of extracellular DNA in river sediment facilitates antibiotic resistance gene propagation. Environmental Science \& Technology, 48(1): 71-78

Martínez J L (2008). Antibiotics and antibiotic resistance genes in natural environments. Science, 321(5887): 365-367

Moriya T, Hikota T, Yumoto I, Ito T, Terui Y, Yamagishi A, Oshima T (2011). Calditerricola satsumensis gen. nov., sp. nov. and Calditerricola yamamurae sp. nov., extreme thermophiles isolated from a high-temperature compost. International Journal of Systematic and Evolutionary Microbiology, 61(3): 631-636

Ngo P T, Rumpel C, Ngo Q A, Alexis M, Vargas G V, Mora Gil M D L L, Dang D K, Jouquet P (2013). Biological and chemical reactivity and phosphorus forms of buffalo manure compost, vermicompost and their mixture with biochar. Bioresource Technology, 148: 401407

Nielsen K M, Johnsen P J, Bensasson D, Daffonchio D (2007a). Release and persistence of extracellular DNA in the environment. Environmental Biosafety Research, 6(1-2): 37-53

Nielsen K M, Johnsen P J, Bensasson D, Daffonchio D (2007b). Release and persistence of extracellular DNA in the environment. Environmental Biosafety Research, 6(1-2): 37-53

O'Neill J (2014). Antimicrobial resistance: Tackling a crisis for the health and wealth of nations. Review on Antimicrobial Resistance, 20: $1-16$

Oshima T, Moriya T (2008). A preliminary analysis of microbial and biochemical properties of high-temperature compost. Annals of the New York Academy of Sciences, 1125(1): 338-344

Peng H, Gu J, Wang X, Wang Q, Sun W, Hu T, Guo H, Ma J, Bao J (2020). Insight into the fate of antibiotic resistance genes and bacterial community in co-composting green tea residues with swine manure. Journal of Environmental Management, 266: 110581

Peng S, Li H, Song D, Lin X, Wang Y (2018). Influence of zeolite and superphosphate as additives on antibiotic resistance genes and bacterial communities during factory-scale chicken manure composting. Bioresource Technology, 263: 393-401

Pruden A, Pei R, Storteboom H, Carlson K H (2006). Antibiotic resistance genes as emerging contaminants: Studies in Northern Colorado. Environmental Science \& Technology, 40(23): 74457450

Qian X, Gu J, Sun W, Wang X, Li H (2019). Effects of passivators on antibiotic resistance genes and related mechanisms during composting of copper-enriched pig manure. Science of the Total Environment, 674: 383-391

Qian X, Gu J, Sun W, Wang X J, Su J Q, Stedfeld R (2018). Diversity, abundance, and persistence of antibiotic resistance genes in various types of animal manure following industrial composting. Journal of Hazardous Materials, 344: 716-722

Ren S, Lu A, Guo X, Zhang Q, Wang Y, Guo X, Wang L, Zhang B (2019). Effects of co-composting of lincomycin mycelia dregs with furfural slag on lincomycin degradation, degradation products, antibiotic resistance genes and bacterial community. Bioresource Technology, 272: 83-91

Su J Q, Wei B, Ou Yang W Y, Huang F Y, Zhao Y, Xu H J, Zhu Y G (2015). Antibiotic resistome and its association with bacterial communities during sewage sludge composting. Environmental Science \& Technology, 49(12): 7356-7363
Sui Q, Chen Y, Yu D, Wang T, Hai Y, Zhang J, Chen M, Wei Y (2019). Fates of intracellular and extracellular antibiotic resistance genes and microbial community structures in typical swine wastewater treatment processes. Environment International, 133: 105183

Sun M, Ye M, Wu J, Feng Y, Wan J, Tian D, Shen F, Liu K, Hu F, Li H, Jiang X, Yang L, Kengara F O (2015). Positive relationship detected between soil bioaccessible organic pollutants and antibiotic resistance genes at dairy farms in Nanjing, Eastern China. Environmental Pollution, 206: 421-428

Tang Z, Xi B, Huang C, Tan W, Li W, Zhao X, Liu K, Xia X (2020). Mobile genetic elements in potential host microorganisms are the key hindrance for the removal of antibiotic resistance genes in industrialscale composting with municipal solid waste. Bioresource Technology, 301: 122723

Vikesland P, Pruden A, Alvarez P, Aga D, Bürgmann H, Li X, Manaia C, Nambi I, Wigginton K, Zhang T, Zhu Y (2017). Toward a comprehensive strategy to mitigate dissemination of environmental sources of antibiotic resistance. Environmental Science \& Technology, 51(22): 13061-13069

Wang B, Zhang Y, Zhu D, Li H (2020a). Assessment of bioavailability of biochar-sorbed tetracycline to Escherichia coli for activation of antibiotic resistance genes. Environmental Science \& Technology, 54: $12920-12928$

Wang F, Stedtfeld R D, Kim O S, Chai B, Yang L, Stedtfeld T M, Hong S G, Kim D, Lim H S, Hashsham S A, Tiedje J M, Sul W J (2016a). Influence of soil characteristics and proximity to Antarctic research stations on abundance of antibiotic resistance genes in soils. Environmental Science \& Technology, 50(23): 12621-12629

Wang J, Ben W, Zhang Y, Yang M, Qiang Z (2015). Effects of thermophilic composting on oxytetracycline, sulfamethazine, and their corresponding resistance genes in swine manure. Environmental Science. Processes \& Impacts, 17(9): 1654-1660

Wang L, Wang J, Wang J, Zhu L, Yang L, Yang R (2019). Distribution characteristics of antibiotic resistant bacteria and genes in fresh and composted manures of livestock farms. Science of the Total Environment, 695: 133781

Wang Q, Gu J, Wang X, Ma J, Hu T, Peng H, Bao J, Zhang R (2020b). Effects of nano-zerovalent iron on antibiotic resistance genes and mobile genetic elements during swine manure composting. Environmental Pollution, 258: 113654

Wang R, Zhang J, Sui Q, Wan H, Tong J, Chen M, Wei Y, Wei D (2016b). Effect of red mud addition on tetracycline and copper resistance genes and microbial community during the full scale swine manure composting. Bioresource Technology, 216: 1049-1057

Wu D, Huang X H, Sun J Z, Graham D W, Xie B (2017). Antibiotic resistance genes and associated microbial community conditions in aging landfill systems. Environmental Science \& Technology, 51 (21): 12859-12867

Xi B, Zhao X, He X, Huang C, Tan W, Gao R, Zhang H, Li D (2016). Successions and diversity of humic-reducing microorganisms and their association with physical-chemical parameters during composting. Bioresource Technology, 219: 204-211

Yin Y, Gu J, Wang X, Song W, Zhang K, Sun W, Zhang X, Zhang Y, Li $H$ (2017). Effects of copper addition on copper resistance, antibiotic resistance genes, and intl1 during swine manure composting. Frontiers in Microbiology, 8: 344 
Youngquist C P, Mitchell S M, Cogger C G (2016). Fate of antibiotics and antibiotic resistance during digestion and composting: a review. Journal of Environmental Quality, 45(2): 537-545

Yu M, He X, Liu J, Wang Y, Xi B, Li D, Zhang H, Yang C (2018). Characterization of isolated fractions of dissolved organic matter derived from municipal solid waste compost. Science of the Total Environment, 635: 275-283

Yu Z, Wu C, Yang G Q, Zhou S G (2015). Planifilum caenisp. nov., a novel member of thermoactinomycete isolated from sludge compost. Current Microbiology, 70(1): 135-140

Yuan Q B, Huang Y M, Wu W B, Zuo P, Hu N, Zhou Y Z, Alvarez P J J (2019). Redistribution of intracellular and extracellular free \& adsorbed antibiotic resistance genes through a wastewater treatment plant by an enhanced extracellular DNA extraction method with magnetic beads. Environment International, 131: 104986

Zammit I, Marano R B M, Vaiano V, Cytryn E, Rizzo L (2020). Changes in antibiotic resistance gene levels in soil after irrigation with treated wastewater: A comparison between heterogeneous photocatalysis and chlorination. Environmental Science \& Technology, 54(12): 7677-7686

Zhang B, Wang M M, Wang B, Xin Y, Gao J, Liu H (2018a). The effects of bio-available copper on macrolide antibiotic resistance genes and mobile elements during tylosin fermentation dregs co-composting. Bioresource Technology, 251: 230-237

Zhang J, Chen M, Sui Q, Tong J, Jiang C, Lu X, Zhang Y, Wei Y (2016a). Impacts of addition of natural zeolite or a nitrification inhibitor on antibiotic resistance genes during sludge composting. Water Research, 91: 339-349

Zhang J, Lin H, Ma J, Sun W, Yang Y, Zhang X (2019a). Compostbulking agents reduce the reservoir of antibiotics and antibiotic resistance genes in manures by modifying bacterial microbiota. Science of the Total Environment, 649: 396-404

Zhang L, Gu J, Wang X, Sun W, Yin Y, Sun Y, Guo A, Tuo X (2017a). Behavior of antibiotic resistance genes during co-composting of swine manure with Chinese medicinal herbal residues. Bioresource Technology, 244(Pt 1): 252-260

Zhang L, Wu Q (2005). Single gene retrieval from thermally degraded DNA. Journal of Biosciences, 30(5): 599-604

Zhang M, He L Y, Liu Y S, Zhao J L, Liu W R, Zhang J N, Chen J, He L K, Zhang Q Q, Ying G G (2019). Fate of veterinary antibiotics during animal manure composting. Science of the Total Environment, 650: 1363-1370

Zhang Q Q, Ying G G, Pan C G, Liu Y S, Zhao J L (2015). Comprehensive evaluation of antibiotics emission and fate in the river basins of China: Source analysis, multimedia modeling, and linkage to bacterial resistance. Environmental Science \& Technology, 49(11): 6772-6782

Zhang R, Gu J, Wang X, Li Y, Zhang K, Yin Y, Zhang X (2018b). Contributions of the microbial community and environmental variables to antibiotic resistance genes during co-composting with swine manure and cotton stalks. Journal of Hazardous Materials, 358: $82-91$

Zhang X, Li J, Fan W Y, Yao M C, Yuan L, Sheng G P (2019b). Enhanced photodegradation of extracellular antibiotic resistance genes by dissolved organic matter photosensitization. Environmental Science \& Technology, 53(18): 10732-10740

Zhang Y, Gu A Z, He M, Li D, Chen J (2017b). Subinhibitory concentrations of disinfectants promote the horizontal transfer of multidrug resistance genes within and across genera. Environmental Science \& Technology, 51(1): 570-580

Zhang Y, Li A, Dai T, Li F, Xie H, Chen L, Wen D (2018c). Cell-free DNA: A neglected source for antibiotic resistance genes spreading from WWTPs. Environmental Science \& Technology, 52(1): 248257

Zhang Y, Li H, Gu J, Qian X, Yin Y, Li Y, Zhang R, Wang X (2016b). Effects of adding different surfactants on antibiotic resistance genes and intI1 during chicken manure composting. Bioresource Technology, 219: 545-551

Zhang Y, Snow D D, Parker D, Zhou Z, Li X (2013). Intracellular and extracellular antimicrobial resistance genes in the sludge of livestock waste management structures. Environmental Science \& Technology, 47(18): 10206-10213

Zhou S, Zhu Y, Yan Y, Wang W, Wang Y (2019). Deciphering extracellular antibiotic resistance genes (eARGs) in activated sludge by metagenome. Water Research, 161: 610-620

Zhu D, Zheng F, Chen Q L, Yang X R, Christie P, Ke X, Zhu Y G (2018). Exposure of a soil collembolan to $\mathrm{Ag}$ nanoparticles and $\mathrm{AgNO}_{3}$ disturbs its associated microbiota and lowers the incidence of antibiotic resistance genes in the gut. Environmental Science \& Technology, 52(21): 12748-12756

Zhu L, Zhao Y, Zhang W, Zhou H, Chen X, Li Y, Wei D, Wei Z (2019a). Roles of bacterial community in the transformation of organic nitrogen toward enhanced bioavailability during composting with different wastes. Bioresource Technology, 285: 121326

Zhu Y G, Johnson T A, Su J Q, Qiao M, Guo G X, Stedtfeld R D, Hashsham S A, Tiedje J M (2013). Diverse and abundant antibiotic resistance genes in Chinese swine farms. Proceedings of the National Academy of Sciences of the United States of America, 110(9): 34353440

Zhu Y G, Zhao Y, Li B, Huang C L, Zhang S Y, Yu S, Chen Y S, Zhang T, Gillings M R, Su J Q (2017). Continental-scale pollution of estuaries with antibiotic resistance genes. Nature Microbiology, 2(4): 16270

Zhu Y G, Zhao Y, Zhu D, Gillings M, Penuelas J, Ok Y S, Capon A, Banwart S (2019b). Soil biota, antimicrobial resistance and planetary health. Environment International, 131: 105059

Zou Y, Tu W, Wang H, Fang T (2020). Anaerobic digestion reduces extracellular antibiotic resistance genes in waste activated sludge: The effects of temperature and degradation mechanisms. Environment International, 143: 105980 